\title{
Median neuropathy at the wrist in patients with systemic sclerosis: two-year follow-up study
}

\author{
Pornhathai T. Sriwong ${ }^{1}$, Patpiya Sirasaporn ${ }^{1}$, Chingching Foochareon ${ }^{2}$, Kannikar Srichompoo $^{1}$ \\ ${ }^{1}$ Department of Rehabilitation Medicine, Faculty of Medicine, Khon Kaen University, Khon Kaen, Thailand \\ ${ }^{2}$ Department of Medicine, Faculty of Medicine, Khon Kaen University, Khon Kaen, Thailand
}

\begin{abstract}
Objectives: To determine the incidence and factors associated with median neuropathy at the wrist (MNW) in systemic sclerosis (SSc) patients using an electrodiagnostic (EDX) study.

Material and methods: SSc patients who attended the scleroderma clinic, Srinagarind Hospital, were prospectively evaluated by questionnaire, physical examination, and EDX study. The questionnaire consisted of the baseline characteristics, type of scleroderma, clinical signs and symptoms associated with scleroderma, and the Boston questionnaire. The physical examinations were the Modified Rodnan Skin Score, motor power grading of bilateral abductor pollicis brevis muscle, and pinprick sensation of median nerve distribution of both hands. Moreover, the EDX study, which was composed of median and ulnar sensory and motor nerve conduction studies of both hands and electromyography of the abductor pollicis brevis muscles, was evaluated at baseline and following two years.

Results: Out of 75 systemic sclerosis patients, 50 individuals participated in the study. Diffuse cutaneous type of SSc is the most common type (72\%). The common SSc associated symptoms were Raynaud phenomenon, skin tightness and hand deformity. Incidence of MNW was 90 cases per 1000 person-years. Following a two-year interval, the percentages of mild, moderate and severe MNW were $28 \%, 22 \%$ and $6 \%$ respectively. Most of those with MNW were still particularly asymptomatic in a mild degree. Moreover, 12 out of 50 patients (24\%) had a worse EDX result. There was no statistically significant association between MNW occurrence and studied factors.

Conclusions: MNW commonly has high incidence in SSc patients. Therefore, SSc patients should be followed up in both clinical and EDX studies for MNW screening. SSc patients with asymptomatic MNW might need clinical follow-up for early diagnosis.
\end{abstract}

Key words: systemic sclerosis, median neuropathy at wrist, electrodiagnostic study.

\section{Introduction}

Median neuropathy at the wrist (MNW), which is the most common peripheral neuropathy, is defined by compression of the median nerve in the wrist. Nerve compression may sequentially create problems relating to lesions at the level of the myelin sheath and at the axonal level, and changes to the supporting connective tissue [1]. There are many predisposing factors of MNW such as congenital bone disease, metabolic diseases including diabetes, degenerative disease, and repetitive trauma, as well as physiological conditions such as pregnancy. All of these are prone to compress the median nerve in the wrist area. The symptoms of MNW are paresthesia, wrist pain, numbness along the distribution of the median nerve in the affected hand, and abductor pollicis brevis muscle weakness [2, 3]. These symptoms affect capability of work performance, emotional well-being and quality of life $[4,5]$.

\section{Address for correspondence:}

Patpiya Sirasaporn, Department of Rehabilitation Medicine, Faculty of Medicine, Khon Kaen University, 123 Mittraparb Rd,

40002 Khon Kaen, Thailand, e-mail: patpiya4@gmail.com

Submitted: 16.07.2018; Accepted: 24.09.2018 
The diagnosis of MNW is composed of history, physical examination, and a provocative test, which are not completely specific to diagnose such disease. At present, electrodiagnostic (EDX) study using specific medical equipment has an important role for MNW diagnosis. EDX is used to determine the definite neurological diagnosis and to document the severity of disease. Regarding MNW, the median nerve conduction study is abnormal whereas the ulnar nerve conduction study is still normal.

Systemic sclerosis (SSc) is a connective tissue disease which is characterized by fibrosis of the skin and multiple internal organs, and vasculopathy [6]. Although SSc is uncommon, it has a high morbidity and mortality. Characteristic features of the disease are skin tightness and internal organ fibrosis. These make skin and internal organs thicker and harder. Thickness of the skin layer around the wrist joint directly compresses the median nerve, which increases the risk of MNW in SSc patients. Moreover, median nerves usually lose their elasticity in such patients. These results suggested that MNW in SSc patients involves directly increased stiffness of the median nerve [7]. Therefore, the most common peripheral neuropathy in SSc patients is MNW, which occurs in about 3.2-44\% [8-13]. The prevalence of MNW in such patients was higher than in the general population, which was found to be about $0.9-10 \%$ [3].

Regarding the literature review, there was only one study of MNW in SSc patients in Thailand, which found that prevalence of MNW was $44 \%$. The percentage of symptomatic MNW was $12 \%$ and that of asymptomatic MNW was $88 \%$ [13]. Moreover, the duration of EDX study changes in MNW usually occurs around two years [14]. Therefore, in this study, EDX study of the bilateral median and ulnar nerves was evaluated at baseline and after two years.

To the best of our knowledge, there are no previous published studies which followed up MNW in SSc patients in a two-year interval. The purpose of the present study was to determine the incidence and factors associated with MNW in SSc patients by using EDX study.

\section{Material and methods Participants}

Systemic sclerosis patients who attended the scleroderma clinic, medicine department, Srinagarind Hospital from October 2013 to August 2014 were recruited to the study. Duration of the study was October 2013 to August 2016. Inclusion criteria were age more than 18 years old, all types of systemic sclerosis patients, and provision of written informed consent. Exclusion criteria were renal failure, pregnancy, diabetes mellitus, hyperthyroidism or hypothyroidism, rheumatoid arthritis, wrist fracture or dislocation, overlapping syndrome, and history of carpal tunnel release surgery.

This study was approved by the Khon Kaen University Ethics Committee for Human Research (HE591304). Written informed consent was obtained from all patients.

\section{Methods}

All participants were assessed by physical examination and EDX study. The first part of the questionnaire consisted of baseline characteristics: age, gender, hand dominance, weight, height and underlying disease. The second part of the questionnaire was composed ofSSc associated data: duration, type, and symptoms of SSc. The last part of the questionnaire was the Thai version of the Boston questionnaire [15], which was the severity rating instrument for carpal tunnel syndrome. It consisted of 11 items of symptom severity scores (SSS) and eight items of functional severity scores (FSS). The questionnaire was presented in multiple-choice format, and scores were assigned from 1 point (mildest) to 5 points (most severe). No response to a certain question was given 0 point. Each score was calculated as the mean of the responses of the individual items. Gradings were divided into five groups according to mean score: extreme (4.1-5 points), severe (3.1-4 points), moderate (2.1-3 points), mild (1.1-2 points) and minimal (0.1-1 point) [16].

Physical examination involved sensation of both hands, motor power of both the abductor pollicis brevis muscles, Tinel's sign, Phalen maneuver and Modified Rodnan Skin Score. Patients with SSc underwent EDX study which was composed of median and ulnar sensory and motor nerve conduction studies of both hands and electromyography of the abductor pollicis brevis muscles. EDX was performed with a Medtronic Keypoint ${ }^{\circledR}$.NET device by the physiatrists. During the EDX study, hand temperature was maintained at $32^{\circ} \mathrm{C}$ or higher.

\section{Electrodiagnostic study}

Electrodiagnostic study was recorded with standardized methods according to the American Association of Neuromuscular and Electrodiagnostic Medicine (AANEM).

1. Sensory nerve conduction study: antidromic technique recording distal sensory latency (DSL) and nerve conduction velocity:

1.1. Median sensory nerve conduction study:

- E1 active: Ring electrode at proximal phalanx of the middle finger, just distal to metacarpal joint.

- E2 reference: Ring electrode at $4 \mathrm{~cm}$ distal to E1.

- Stimulation site: At wrist (about $14 \mathrm{~cm}$ proximal to the active electrode at E1) and elbow region. 
1.2. Ulnar sensory nerve conduction study:

- E1 active: Ring electrode at proximal phalanx of the little finger.

- E2 reference: Ring electrode at $4 \mathrm{~cm}$ distal to E1.

- Stimulation site: At wrist (about $14 \mathrm{~cm}$ proximal to the active electrode at E1) and elbow region.

2. Motor nerve conduction study: orthodromic technique recording distal motor latency (DML), compound muscle action potential (CMAP) amplitude and nerve conduction velocity:

2.1. Median motor nerve conduction study:

- E1 active: surface electrode at middle point of abductor pollicis brevis muscle.

- E2 reference: surface electrode at distal interphalangeal joint of thumb.

- Stimulation site: At wrist (about $8 \mathrm{~cm}$ proximal to the active electrode at E1) and elbow region.

2.2. Ulnar motor nerve conduction study:

- E1 active: surface electrode at middle point of abductor digiti minimi muscle.

- E2 reference: surface electrode at distal interphalangeal joint of the little finger.

- Stimulation site: At wrist (about $8 \mathrm{~cm}$ proximal to the active electrode at E1) and elbow region

3. Electromyography (EMG):

3.1. Needle examination: If the CMAP amplitude of median nerve is less than 5 millivolts, needle EMG of abductor pollicis brevis muscle will be done.

Electrodiagnostic (EDX) of MNW was obtained according to normative values and an abnormality was defined according to AANEM [17]. MNW was classified in three stages.

- Mild: only prolonged DSL (more than 3.50 milliseconds).

- Moderate: prolonged DSL (more than 3.50 milliseconds) and prolonged DML (more than 4.2 milliseconds).

- Severe: absent or prolonged DSL (more than 3.50 milliseconds) and DML (more than 4.2 milliseconds) and decreased compound muscle action potential (CMAP) and abnormality in electromyography.

Electrodiagnostic study was evaluated at baseline and after two years. The individual EDX results at two years were compared with the baseline results and were classified in three levels.

- Improvement: EDX study showed better results. For example, first, the baseline EDX study found MNW but after two years the EDX study result found no evidence of MNW. Secondly, after two years the EDX study result found improvement of MNW stages.

- Constancy: EDX study showed unchanged results. For example, first, the EDX study result at both baseline and two years found the same stages of MNW.
Secondly, the EDX study result at both baseline and two years showed no evidence of MNW.

- Deterioration: EDX study showed worsened results. For example, first, the baseline EDX study result found no evidence of MNW but after two years the EDX study result found MNW. Secondly, after two years the EDX study result found MNW regression stages.

\section{Statistical analysis}

Data from all participants were analyzed according to data being available. No imputation was implemented to missing data. All statistical tests were two-sided with a significant level of 0.05. All statistical analyses were performed using SPSS 19.0 (IBM SPSS Statistics version 19). The continuous data were presented as mean and standard deviation (SD). The ordinal data were shown as frequency and percentage. Prevalence of MNW at baseline and two years was presented as a percentage. Incidence of MNW was reported as cases per 1000 person-years. Associations between factors and MNW were analyzed by univariate analysis (significant $p$-value $<0.05)$.

\section{Results}

Out of 75 SSc participants, 25 subjects dropped out: 1 refused to repeat the EDX study, 9 died and 15 were lost to follow-up. The number of completed participants was 50, out of whom 39 (78\%) were female. The average age of the participants was $53 \pm 11$ years (range 24-78 years). The body mass index of most the participants was underweight. Duration of systemic sclerosis disease was $10.1 \pm 5.2$ years. Diffuse cutaneous type of SSc is the most common type (72\%). The common SSc associated symptoms were Raynaud phenomenon, skin tightness and hand deformity. The Boston questionnaire showed that most of the participants presented with lower severity of MNW (74\%) and also a lower degree of difficulty in hand functioning (48\%). The percentage of MNW-related symptoms such as abductor pollicis brevis weakness and/or numbness along the median nerve distribution was 18\% (Table I).

According to the EDX study, following a two-year interval, incidence of MNW was 90 cases per 1000 person-years. The percentages of mild, moderate and severe MNW were $28 \%, 22 \%$ and $6 \%$ respectively (Table II). At baseline, most of the participants with MNW were especially asymptomatic in a mild degree (Fig. 1). Following a two-year interval, most of those with MNW were still particularly asymptomatic in a mild degree. Moreover, those with a moderate degree of asymptomatic MNW were similar to symptom- 
Table I. Baseline characteristics

\begin{tabular}{|c|c|c|}
\hline Data & $\begin{array}{l}\text { Number } \\
(N=50)\end{array}$ & Percentage \\
\hline $\begin{array}{l}\text { Age (years) } \\
\text { mean } \pm S D\end{array}$ & $\begin{array}{c}53 \pm 11 \\
(24-78)\end{array}$ & \\
\hline \multicolumn{3}{|l|}{ Gender } \\
\hline Female & 39 & 78 \\
\hline \multicolumn{3}{|l|}{ Hand dominance } \\
\hline Right & 48 & 96 \\
\hline \multicolumn{3}{|l|}{ Body mass index $\left(\mathrm{kg} / \mathrm{m}^{2}\right)$} \\
\hline Underweight & 18 & 36 \\
\hline Normal weight & 14 & 28 \\
\hline Overweight & 7 & 14 \\
\hline Obesity & 11 & 22 \\
\hline \multicolumn{3}{|l|}{ Underlying disease } \\
\hline Hypertension & 8 & 16 \\
\hline Dyslipidemia & 4 & 8 \\
\hline Others & 2 & 4 \\
\hline $\begin{array}{l}\text { Duration of systemic sclerosis } \\
\text { (years) mean } \pm S D\end{array}$ & $10.1 \pm 5.2$ & \\
\hline \multicolumn{3}{|l|}{ Systemic sclerosis (SSc) type } \\
\hline Limited cutaneous SSC & 14 & 28 \\
\hline Diffused cutaneous SSC & 36 & 72 \\
\hline $\begin{array}{l}\text { Skin thickness } \\
\text { Mean } \pm \text { SD }\end{array}$ & $0.7 \pm 1.4$ & \\
\hline \multicolumn{3}{|l|}{ SSc symptoms } \\
\hline Raynaud phenomenon & 30 & 60 \\
\hline Ulcer & 11 & 22 \\
\hline Telangiectasia & 16 & 32 \\
\hline Calcinosis & 3 & 6 \\
\hline Salt and pepper skin changes & 15 & 30 \\
\hline Skin edema & 2 & 4 \\
\hline Skin tightness & 17 & 34 \\
\hline Tendon rub & 8 & 16 \\
\hline Hand deformity & 17 & 34 \\
\hline Arthritis & 1 & 2 \\
\hline Dysphagia & 16 & 32 \\
\hline Heartburn & 16 & 32 \\
\hline Dyspepsia & 9 & 18 \\
\hline \multicolumn{3}{|l|}{ Boston questionnaire } \\
\hline \multicolumn{3}{|l|}{ Severity } \\
\hline Least & 6 & 12 \\
\hline Less & 37 & 74 \\
\hline Moderate & 7 & 14 \\
\hline Severe & 0 & 0 \\
\hline Very severe & 0 & 0 \\
\hline
\end{tabular}

\begin{tabular}{|lcc|}
\hline Data & $\begin{array}{c}\text { Number } \\
(N=50)\end{array}$ & Percentage \\
\hline Loss of hand function & 21 & 42 \\
\hline Least & 24 & 48 \\
\hline Less & 5 & 10 \\
\hline Moderate & 0 & 0 \\
\hline Severe & 0 & 0 \\
\hline Very severe & 9 & 18 \\
\hline $\begin{array}{l}\text { Abductor pollicis brevis weak- } \\
\text { ness and/or numbness along } \\
\text { the median nerve distribution }\end{array}$ & & \\
\hline \begin{tabular}{l} 
Special test \\
\hline Tinel's sign positive
\end{tabular} & 2 & 4 \\
\hline Phalen maneuver positive & 5 & 10 \\
\hline
\end{tabular}

Table II. Electrodiagnostic study

\begin{tabular}{|lcc|}
\hline $\begin{array}{l}\text { EDX results } \\
\begin{array}{c}\text { At baseline } \\
(N=50) \\
n(\%)\end{array}\end{array}$ & $\begin{array}{c}\text { After 2 years } \\
(N=50) \\
n(\%)\end{array}$ \\
\hline Median neuropathy at wrist & $14(28)$ \\
\hline Mild & $13(26)$ & $11(22)$ \\
\hline Moderate & $6(12)$ & $3(6)$ \\
\hline Severe & $4(8)$ & $28(56)$ \\
\hline All MNW & $23(46)$ & $22(44)$ \\
\hline Normal & $27(54)$ & \\
\hline
\end{tabular}

atic MNW and all of those with a severe degree of MNW were symptomatic (Fig. 2).

According to SSc type, the percentage of patients with MNW in diffuse SSc type was 38\%, whereas the percentage of occurrence of MNW in limited SSc type was $18 \%$. Regarding comparison of the EDX study from baseline to two years later, most EDX results were constant (66\%). Participants with no evidence of MNW still have no verification of MNW (40\%) and participants with evidence of severe MNW still have verification of severe MNW (6\%). In the deterioration group, most of the participants with no evidence of MNW regressed to a mild degree of MNW (12\%), whereas in the improvement group, only $4 \%$ of those with a mild degree of MNW returned to no evidence of MNW (Table III). Moreover, there were no statistically significant associations between MNW occurrence and demographic and clinical factors (Table IV). 


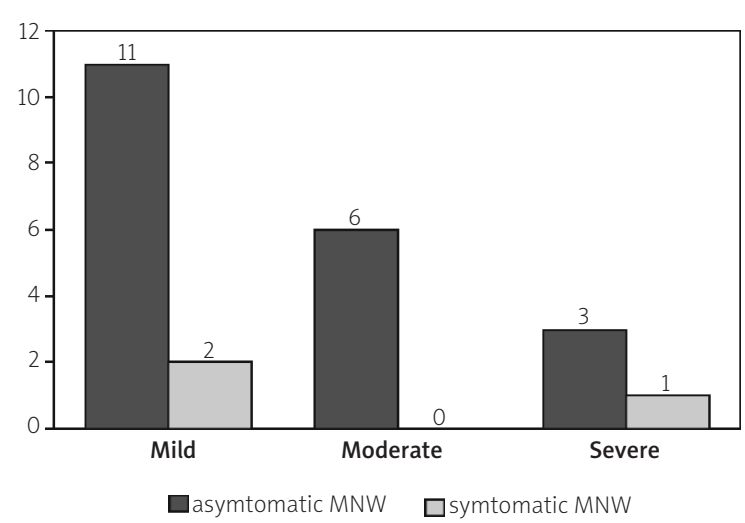

Fig. 1. Electrodiagnostic study at baseline.

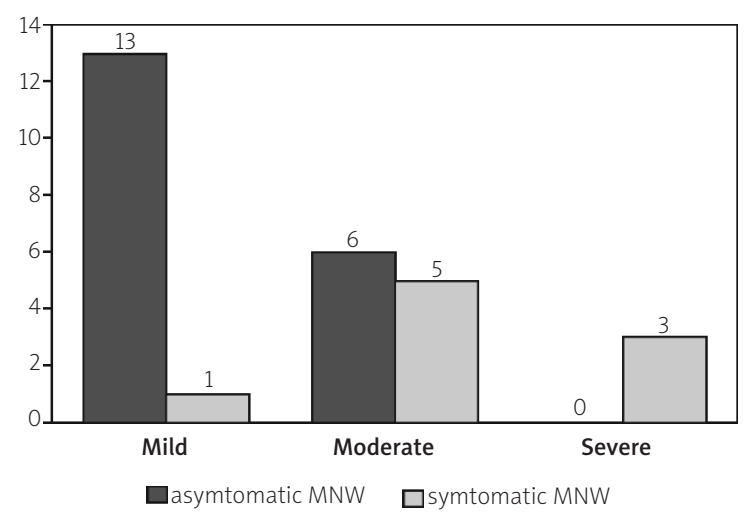

Fig. 2. Electrodiagnostic study at 2 years.

Table III. Electrodiagnostic study classification

\begin{tabular}{|c|c|c|c|}
\hline \multirow{2}{*}{$\begin{array}{l}\text { Changes of } \\
\text { MNW severity }\end{array}$} & \multicolumn{2}{|c|}{ MNW severity } & \multirow[t]{2}{*}{$n(\%)$} \\
\hline & At baseline & After 2 years & \\
\hline \multirow[t]{4}{*}{ Improvement } & severe & moderate & $1(2)$ \\
\hline & moderate & mild & $2(4)$ \\
\hline & mild & none & $2(4)$ \\
\hline & \multicolumn{2}{|c|}{ total } & $5(10)$ \\
\hline \multirow[t]{5}{*}{ Constancy } & severe & severe & $3(6)$ \\
\hline & moderate & moderate & $4(8)$ \\
\hline & mild & mild & $6(12)$ \\
\hline & none & none & $20(40)$ \\
\hline & \multicolumn{2}{|c|}{ total } & $33(66)$ \\
\hline \multirow[t]{4}{*}{ Deterioration } & mild & moderate & $5(10)$ \\
\hline & none & moderate & $1(2)$ \\
\hline & none & mild & $6(12)$ \\
\hline & \multicolumn{2}{|c|}{ total } & $12(24)$ \\
\hline
\end{tabular}

Table IV. Association between demographic and clinical factors and median neuropathy at wrist

\begin{tabular}{|c|c|c|c|}
\hline Factors & $\begin{array}{c}\text { MNW } \\
(n)\end{array}$ & $\begin{array}{c}\text { No MNW } \\
(n)\end{array}$ & $p$-value \\
\hline \multicolumn{4}{|l|}{ Age (years) } \\
\hline$<50$ & 7 & 10 & \multirow{2}{*}{0.147} \\
\hline$\geq 50$ & 21 & 12 & \\
\hline \multicolumn{4}{|l|}{ Gender } \\
\hline Male & 7 & 4 & \multirow{2}{*}{0.734} \\
\hline Female & 21 & 18 & \\
\hline \multicolumn{4}{|c|}{ Hand dominance } \\
\hline Right & 19 & 14 & \multirow{2}{*}{0.773} \\
\hline Left & 9 & 8 & \\
\hline \multicolumn{4}{|l|}{$\mathrm{BMI}$} \\
\hline Normal & 17 & 18 & \multirow{2}{*}{0.095} \\
\hline Obesity & 11 & 4 & \\
\hline \multicolumn{4}{|c|}{ Systemic sclerosis type } \\
\hline Limited & 9 & 5 & \multirow{2}{*}{0.340} \\
\hline Diffused & 19 & 17 & \\
\hline \multicolumn{4}{|c|}{ Duration of systemic sclerosis (years) } \\
\hline$<5$ & 4 & 0 & \multirow{2}{*}{0.121} \\
\hline$\geq 5$ & 22 & 24 & \\
\hline \multicolumn{4}{|c|}{ Raynaud phenomenon } \\
\hline negative & 9 & 9 & \multirow{2}{*}{0.565} \\
\hline positive & 19 & 13 & \\
\hline \multicolumn{4}{|c|}{ Skin tightness } \\
\hline negative & 19 & 14 & \multirow{2}{*}{0.773} \\
\hline positive & 9 & 8 & \\
\hline \multicolumn{4}{|l|}{ Skin ulcer } \\
\hline negative & 20 & 18 & \multirow{2}{*}{0.512} \\
\hline positive & 8 & 4 & \\
\hline \multicolumn{4}{|c|}{ Hand deformity } \\
\hline negative & 19 & 14 & \multirow{2}{*}{0.773} \\
\hline positive & 9 & 8 & \\
\hline \multicolumn{4}{|c|}{ Telangiectasia } \\
\hline negative & 21 & 13 & \multirow{2}{*}{0.360} \\
\hline positive & 7 & 9 & \\
\hline \multicolumn{4}{|l|}{ Skin edema } \\
\hline negative & 26 & 21 & \multirow{2}{*}{1.000} \\
\hline positive & 2 & 1 & \\
\hline \multicolumn{4}{|c|}{ Salt and pepper skin changes } \\
\hline negative & 18 & 17 & \multirow{2}{*}{0.367} \\
\hline positive & 10 & 5 & \\
\hline
\end{tabular}




\section{Discussion}

In this study, we found a high incidence of MNW in SSc patients compared to the general population, which was 2.76 cases per 1000 person-years [18]. Moreover, the incidence of MNW in SSc patients was higher than the MNW in rheumatoid arthritis patients, which was found to be 4.18 cases per 1000 person-years [19], and the MNW in community-based patients with type 2 diabetes, which was only about 5.5 cases per 1000 person-years [20]. It was in accordance with the hypothesis that fibrosis thickness of the skin layer and swelling around the wrist joint are directly vulnerable to compression of the median nerve [7]. Moreover, Raynaud phenomenon was the main cause of blood vessel constriction that induced coldness or numbness in fingers [7, 12, 21]. In addition, regarding nerve elasticity, SSc patients had stiffer nerves than either healthy people or those other diseases with MNW [7].

Patients with rheumatoid arthritis, diabetes mellitus, hyperthyroidism or hypothyroidism, wrist fracture or dislocation, and with overlapping syndromes were excluded from the present study to eliminate confounding factors because those diseases can affect the median nerve, which may directly cause MNW [1, 2]. However, thyroid dysfunctions are underdiagnosed in SSc patients [22]. It is almost impossible to eliminate all patients with SSc and subclinical hypothyroidism. Therefore, it should be considered that hypothyroidism may be one of the possible causes of MNW in this study.

Among the participants in this study, the most common type of MNW was asymptomatic, which was similar to the previous studies $[8,10]$. Although most of the MNW cases were subclinical, which did not directly affect the routine activity of daily living in SSc patients, a manifestation sign of increased vulnerability to the expression of underlying diffuse damage to the peripheral nervous system was hypothesized. There was evidence that idiopathic MNW in SSc slowly progresses over time and this can be measured with EDX [9]. However, all of the cases with a severe degree of MNW were symptomatic after a 2-year interval. Therefore, SSc patients with MNW-related symptoms such as abductor pollicis brevis weakness and/or numbness along the median nerve distribution should undergo EDX tests to determine the severity of MNW. In the current study, most of the participants with no evidence of MNW regressed to a mild degree of MNW after two years, so it may be caused by increasing age or progression of systemic sclerosis signs and symptoms.

There was no association between factors of systemic sclerosis and MNW, which was in accordance with a previous study [13]. It may be caused by limitation of the sample size, which was mainly calculated for estimating the incidence. Nevertheless, the previous study showed a relationship between gender and incidence of MNW, and it has been suggested that MNW may be considered as an early symptom of rapidly progressive SSc in men [23].

\section{Conclusions}

The present study confirms that MNW commonly occurred in SSc patients. Therefore, SSc patients should be followed up in both clinical and EDX studies for MNW screening. SSc patients with asymptomatic MNW might need clinical follow-up for early diagnosis. In addition, the next cohort study about the results of MNW treatment in SSc patients should be considered.

This study was granted by the Faculty of Medicine Research Fund (IN60343).

The authors declare no conflict of interest.

\section{References}

1. Chammas M, Boretto J, Burmann LM, et al. Carpal tunnel syndrome - Part I (anatomy, physiology, etiology and diagnosis). Rev Bras Ortop 2014; 49: 429-436.

2. Werner RA, Andary M. Carpal tunnel syndrome: pathophysiology and clinical neurophysiology. Clin Neuro Physiol 2002; 113: 13731381.

3. Wahab KW, Sanya EO, Adebayo PB, et al. Carpal Tunnel Syndrome and Other Entrapment Neuropathies. Oman Med J 2017; 32: 449-454.

4. Pogorzelski R, Kulakowska A, Halicka D, et al. Neurological and emotional profile of carpal tunnel syndrome patients. Przegl Lek 2011; 68: 269-273.

5. Katz JN, Lew RA, Bessette L, et al. Prevalence and predictors of long-term work disability due to carpal tunnel syndrome. Am J Ind Med 1998; 33: 543-550.

6. Fett N. Scleroderma: nomenclature, etiology, pathogenesis, prognosis, and treatments: facts and controversies. Clin Dermatol 2013; 31: 432-437.

7. Yagci I, Kenis-Coskun O, Ozsoy T, et al. Increased stiffness of median nerve in systemic sclerosis. BMC Musculoskelet Disord 2017; 18: 1-5.

8. Machet L, Vaillant L, Machet MC, et al. Carpal tunnel syndrome and systemic sclerosis. Dermatology (Basel) 1992; 185: 101-103.

9. Amaral TN, Peres FA, Lapa AT, et al. Neurologic involvement in scleroderma: A systematic review. Semin Arthritis Rheum 2013; 43: 335-347.

10. Mondelli M, Romano C, Della Porta PD, et al. Electrophysiological evidence of "nerve entrapment syndromes" and subclinical peripheral neuropathy in progressive systemic sclerosis (scleroderma). J Neurol 1995; 242: 185-194.

11. Poncelet AN, Connolly MK. Peripheral neuropathy in scleroderma. Muscle Nerve 2003; 28: 330-335. 
12. Lee P, Bruni J, Sukenik S. Neurological manifestations in systemic sclerosis (scleroderma). J Rheumatol 1984; 11: 480-483.

13. Nimitbancha T, Sirasaporn P, Wattanapan P, et al. The prevalence of median neuropathy at wrist in systemic sclerosis patients at Srinagarind Hospital. J Sci Soc 2015; 42: 180-184.

14. Suchtelen M, Becker S, Gruber J, et al. Progression of Carpal Tunnel Syndrome According to Electrodiagnostic Testing in Nonoperatively Treated Patients. Arch Bone Jt Surg 2014; 2 185-191.

15. Upatham S, Kumnerddee W. Reliability of Thai version Boston questionnaire. J Med Assoc Thai 2008; 91: 1250-1256.

16. Rao BH, Kutub M, Patil SD. Carpal tunnel syndrome: Assessment of correlation between clinical, neurophysiological and ultrasound characteristics. J Sci Soc 2012; 39: 124-129.

17. American Association of Electrodiagnostic Medicine, American Academy of Neurology, and American Academy of Physical Medicine and Rehabilitation. Practice parameter for electrodiagnostic studies in carpal tunnel syndrome: summary statement. Muscle Nerve 2002; 25: 918-922.
18. Mondelli M, Giannini F, Giacchi M. Carpal tunnel syndrome incidence in a general population. Neurology 2002; 58: 289294.

19. Lee K-H, Lee C-H, Lee B-G, et al. The incidence of carpal tunnel syndrome in patients with rheumatoid arthritis. Int J Rheum Dis 2015; 18: 52-57.

20. Makepeace A, Davis WA, Bruce DG, et al. Incidence and determinants of carpal tunnel decompression surgery in type 2 diabetes: The Fremantle Diabetes Study. Diabetes Care 2008; 31: 498-500.

21. Lori S, Matucci-Cerinic M, Casale R, et al. Peripheral nervous system involvement in systemic sclerosis: the median nerve as target structure. Clin Exp Rheumatol 1996; 14: 601-605.

22. Kucharz EJ. Thyroid disorders in patients with progressive systemic sclerosis: A review. Clin Rheum 1993; 12: 159-161.

23. Widuchowska M, Kotulska A, Kopeć-Medrek M, et al. Carpal tunnel syndrome as the early symptom of rapidly progressive systemic sclerosis in men. Wiad Lek 2010; 63: 33. 